\title{
Asymptotics toward rarefaction waves and vacuum for 1-d Compressible Navier-Stokes equations
}

\author{
Misha Perepelitsa
}

June 19, 2008

\begin{abstract}
In this work we study time asymptotic behavior of solutions of 1-d Compressible Navier-Stokes equations toward solutions of Euler equations that consist of two rarefaction waves separated by a vacuum region. The analysis relies on various energy estimates.
\end{abstract}

\subsection{Summary and the main result}

Time asymptotic toward rarefaction waves for Compressible Navier-Stokes equations for barotropic fluids was studied in [2,3]. It was proved that if $V^{e}(x / t), U^{e}(x / t)$ is a solution of Euler equations

$$
\left\{\begin{array}{l}
v_{t}=u_{x} \\
u_{t}=-p(v)_{x} \\
p=(v)^{-\gamma}, \gamma \geq 1 \\
(t, x) \in \mathbb{R}^{+} \times \mathbb{R}
\end{array}\right.
$$

with Riemann initial data

$$
\left(v_{r}, u_{r}\right)= \begin{cases}\left(V^{+}, U^{+}\right), & x>0 \\ \left(V^{-}, U^{-}\right), & x<0\end{cases}
$$

which consists of one or two rarefaction waves and such that

$$
\sup _{s} V^{e}(s)<\infty
$$

then, the solution $v, u$ of Compressible Navier-Stokes equations

$$
\left\{\begin{array}{l}
v_{t}=u_{x} \\
u_{t}-\left(\frac{1}{v} u_{x}\right)_{x}+p(v)_{x}=0 \\
p(v)=v^{\gamma}, \gamma \geq 1 \\
(t, x) \in \mathbb{R}^{+} \times \mathbb{R}
\end{array}\right.
$$

with the initial data $\left(v_{0}, u_{0}\right)$ such that

$$
\lim _{x \rightarrow \pm \infty}\left(v_{0}(x), u_{0}(x)\right)=\left(V^{ \pm}, U^{ \pm}\right),
$$


modulo some smoothness conditions, converges, uniformly in space, to the solution of Euler equations:

$$
\lim _{t \rightarrow \infty} \sup _{\mathbb{R}}\left|(v(t, \cdot), u(t, \cdot))-\left(V^{e}(\cdot / t), U^{e}(\cdot / t)\right)\right|=0 .
$$

Condition (2) excludes, in particular, solutions with a vacuum region. In this work we study solutions of (3) with the limits at infinity $\left(V^{ \pm}, U^{ \pm}\right)$leading to a vacuum region separating two rarefaction waves in the corresponding solution of Euler equations. Note, that in this case we can not expect (4) to hold since the velocity in the solution of the Navier-Stokes equation is smooth, but $U^{e}(x / t)$ is discontinuous at $x=0$.

Without finding the form of the asymptotics of $v, u$ uniformly in space we show that the solution converges to the corresponding solution of the Euler equations along characteristics $x / t=$ const., as described in Theorem 1 . To prove it we use energy estimates and make use of an alternative form of the Navier-Stokes equations, see the proof of Lemma 1:

$$
\left\{\begin{array}{l}
v_{t}=w_{x}+(\ln v)_{x x} \\
w_{t}=-p(v)_{x}
\end{array}\right.
$$

\subsubsection{The result}

Let $(v, u)=\left(V^{e}, U^{e}\right)$ be the solution of Euler equations (1) with the Riemann initial data:

$$
\left(v_{r}, u_{r}\right)= \begin{cases}\left(V^{+}, U^{+}\right), & x>0 \\ \left(V^{+},-U^{+}\right), & x<0 \\ V^{+}>0, U^{+}>0 . & \end{cases}
$$

The solution consists of two, symmetric with respect to $x=0$ - axis, rarefaction waves, possibly containing vacuum in between, see subsection 0.3 for formulas. Let

$$
\Phi(v, V)=p(V)(v-V)-\int_{V}^{v} p(s) d s .
$$

For a fixed $V>0, \Phi(v, V)$ is a non-negative, convex function defined on $(0,+\infty)$. Let $\left(v_{0}, u_{0}\right)$ be a pair of smooth, bounded functions on $\mathbb{R}$ such that

$$
\begin{array}{r}
\left.\lim _{x \rightarrow \pm \infty}\left(v_{0}(x), u_{0}(x)\right)\right)=\left(V^{+}, \pm U^{+}\right), \\
\inf _{\mathbb{R}} v_{0}(x)>0, \\
\int\left|u_{0}-u_{r}\right|^{2}<\infty, \quad \int\left|u_{0}-\left(\ln v_{0}\right)_{x}-u_{r}\right|^{2}<\infty, \\
\int \Phi\left(v_{0}, V^{+}\right)<\infty, \\
v_{0, x}, u_{0, x} \in H^{1}(\mathbb{R}) .
\end{array}
$$


Let $(v(t, x), u(t, x))$ be a smooth solution of the Naiver-Stokes equations (3) with initial conditions $v_{0}, u_{0}$. It has the following properties:

$$
\begin{array}{r}
\lim _{x \rightarrow \pm \infty}(v(t, x), u(t, x))=\left(V^{+}, \pm U^{+}\right), \\
c^{-1}(T)<v(t, x)<c(T), \quad t \in(0, T), \\
\int_{0}^{T} \int \frac{1}{v}\left|u_{x}\right|^{2} d x d t \leq c(T), \\
\sup _{t \in(0, T)} \int \Phi\left(v(t, x), V^{+}\right)+\left|u(t, x)-u_{0}\right|^{2} d x \leq c(T),
\end{array}
$$

for any $T>0$ and some $c(T)>0$. Such solution exists as can be shown by methods of [1]. Finally, let

$$
w(t, x)=-\int_{0}^{t} p(v(\tau, x))_{x} d \tau+u_{0}(x)-\left(\ln v_{0}(x)\right)_{x} .
$$

Theorem 1. Let $\gamma>2$,

$$
u_{0, x} \geq 0
$$

and

$$
u_{0, x} \in L^{\frac{2(\gamma-1)}{\gamma-2}}(\mathbb{R}) .
$$

Then, functions $v^{t}(y)=v(t, t y), u^{t}(y)=u(t, t y)$ and $w^{t}(y)=w(t, t y)$ verify the following asymptotic properties as $t \rightarrow+\infty$.

$$
\begin{array}{r}
\int_{\mathbb{R}} \Phi\left(v^{t}(y), V^{e}(y)\right)+\left|w^{t}(y)-U^{e}(y)\right|^{2} d y \rightarrow 0, \\
u^{t}(\cdot) \rightarrow U^{e}(\cdot) i n H^{-1}(I), \\
\forall \epsilon>0, \quad \text { meas }\left((t, \infty) \cap\left\{t: \int_{\mathbb{R}} \frac{\left|u^{t}-w^{t}\right|^{2}}{\left(v^{t}\right)^{\gamma+1}} d y>\epsilon\right\}\right) \rightarrow 0,
\end{array}
$$

where $I$ is any finite interval. Let $\gamma \geq 3 / 2$ and

$$
u_{0, x} \geq 0, \quad u_{0, x}-\left(\ln v_{0}\right)_{x x} \geq 0, \text { a.e. } x \in \mathbb{R} .
$$

Then, properties (17)-(19) hold as well.

Remark 1. The above result holds also for solutions corresponding to less regular, discontinuous, initial data $\left(v_{0}, u_{0}\right)$, by a suitable approximation argument. The condition of the symmetry of the solution of Euler equations is not essential and can be avoided altogether with the minor changes to the proof.

Remark 2. As a byproduct of (17) and (19) it can be shown that

$$
u^{t}(y) \rightarrow U^{e}(y), \quad \text { a.e. } y \in \mathbb{R} .
$$




\subsection{The proof}

The proof is given below in two Lemmas.

Lemma 1. For any $t>t_{0}>0$ it holds:

$$
\begin{aligned}
& \int\left(\Phi\left(v(t, \cdot), V^{e}(t, \cdot)\right)+\frac{\left|w(t, \cdot)-U^{e}(t, \cdot)\right|^{2}}{2}\right) \\
&-\int_{t_{0}}^{t} \int \frac{p^{\prime}(v)}{v}\left|v_{x}\right|^{2} \leq \int\left(\Phi\left(v\left(t_{0}, \cdot\right), V^{e}\left(t_{0}, \cdot\right)\right)+\frac{\left|w\left(t_{0}, \cdot\right)-U^{e}\left(t_{0}, \cdot\right)\right|^{2}}{2}\right) \\
&+\int_{t_{0}}^{t} \int \frac{v_{x}(\tau, x)}{v(\tau, x)} p\left(V^{e}(\tau, x)\right)_{x} d x d \tau,
\end{aligned}
$$

provided that

$$
\left(w_{0}(x)-U^{e}(0, x)\right) \in L^{2}(\mathbb{R}),
$$

where

$$
w(t, x)=\int_{0}^{t} p(v(\tau, x))_{x} d \tau+u_{0}(x)-\left(\ln v_{0}\right)_{x}(x) .
$$

and

$$
w_{0}(\cdot)=w(0, \cdot)
$$

Proof. By integrating both equations in (3) in time we get:

$$
\ln v-\ln v_{0}=\int_{0}^{t} v^{-1} u_{x}, \quad u=u_{0}-\int_{0}^{t} p(v)_{x}+\left(\int_{0}^{t} v^{-1} u_{x}\right)_{x} .
$$

Substituting the diffusion term in the second equation with the expression from the first equation and differentiating the result in $x$ we get

$$
u_{x}=u_{0, x}-\left(\int_{0}^{t} p(v)\right)_{x x}+\left(\ln v-\ln v_{0}\right)_{x x}
$$

Using this expression in the first equation of (3) we get

$$
v_{t}=\left(-\int_{0}^{t} p(v)_{x}+u_{0}-\left(\ln v_{0}\right)_{x}\right)_{x}+(\ln v)_{x x} .
$$

Thus, we obtained an alternative form of Navier-Stokes equations in which $w$ plays the role of velocity:

$$
\left\{\begin{array}{l}
v_{t}=w_{x}+(\ln v)_{x x} \\
w_{t}=-p(v)_{x}
\end{array}\right.
$$

Now, for $t>0$ and $x \neq 0$ compute from (7) the expression:

$$
\begin{aligned}
\Phi\left(v, V^{e}\right)_{t}= & \Phi_{v} v_{t}+\Phi_{V} V_{t}^{e} \\
& =-\left(p(v)-p\left(V^{e}\right)\right)\left(v_{t}-V_{t}^{e}\right)-\underbrace{\left(p(v)-p\left(V^{e}\right)-p^{\prime}\left(V^{e}\right)\left(v-V^{e}\right)\right) V^{e} t}_{=: A} .
\end{aligned}
$$


Note, that since $p$ is convex and $V_{t}^{e} \geq 0$, term $A \geq 0$ as well. Using the first equation of (5) and $V_{t}^{e}=U^{e}{ }_{x}$ in the last formula we obtain:

$$
\begin{aligned}
\Phi_{t}=-\left(p(v)-p\left(V^{e}\right)\right)\left(w_{x}-U^{e}{ }_{x}+(\ln v)_{x x}\right)-A \\
=-\left[\left(p(v)-p\left(V^{e}\right)\right)\left(w-U^{e}\right)\right]_{x}+\left(p(v)-p\left(V^{e}\right)\right)_{x}\left(w-U^{e}\right)-A \\
\quad-\left(\frac{p(v)}{v} v_{x}\right)_{x}+\frac{p^{\prime}(v)}{v}\left|v_{x}\right|^{2}+\left(\frac{p\left(V^{e}\right)}{v} v_{x}\right)_{x}-\frac{v_{x}}{v} p\left(V^{e}\right)_{x} .
\end{aligned}
$$

Multiplying the difference of the second equation in (5) and $U^{e}{ }_{t}=-p\left(V^{e}\right)_{x}$ by $w-U^{e}$ we get

$$
\partial_{t} \frac{\left|w-U^{e}\right|^{2}}{2}=-\left(p(v)-p\left(V^{e}\right)\right)_{x}\left(w-U^{e}\right)
$$

Adding (24) and (25) we obtain

$$
\begin{aligned}
\partial_{t}[\Phi & \left.+\frac{\left|w-U^{e}\right|^{2}}{2}\right]+A-\frac{p^{\prime}(v)}{v}\left|v_{x}\right|^{2} \\
& =-\left[\left(p(v)-p\left(V^{e}\right)\right)\left(w-U^{e}\right)\right]_{x}-\left(\frac{p(v)}{v} v_{x}\right)_{x}+\left(\frac{p\left(V^{e}\right)}{v} v_{x}\right)_{x}-\frac{v_{x}}{v} p\left(V^{e}\right)_{x} .
\end{aligned}
$$

Note, that $v, w$ are smooth functions of $t, x$ and $V^{e}, U^{e}$ are continuous except the line $x=0$, but on this line

$$
\begin{gathered}
U^{e}(t, 0 \pm)= \pm U_{0} \\
p\left(V^{e}(t, 0 \pm)\right)=0 .
\end{gathered}
$$

Integrating the above equation in $t, x \in\left(t_{0}, t\right) \times(0,+\infty)$ and $t, x \in\left(t_{0}, t\right) \times(-\infty, 0)$ and adding the result we obtain after introducing the notation

$$
E(t)=\int\left(\Phi\left(v(t, x), V^{e}(t, x)\right)+\frac{\left|w(t, x)-U^{e}(t, x)\right|^{2}}{2}\right) d x
$$

that

$$
E(t)+\int_{t_{0}}^{t} \int A+\int_{t_{0}}^{t} \int \frac{p^{\prime}(v)}{v}\left|v_{x}\right|^{2}=E(0)-\int_{t_{0}}^{t} \int \frac{v_{x}}{v} p\left(V^{e}\right)_{x} \underbrace{-\int_{t_{0}}^{t} 2 U_{0} p(v(0, \tau)) d \tau}_{<0} .
$$

The statement of the lemma follows.

Lemma 2. Assume that

$$
u_{0, x} \geq 0 .
$$

Then, for any $\gamma>2$ there is $c=c(\gamma)$ such that

$$
\sup _{\mathbb{R}}|\ln v(t, \cdot)| \leq c \ln (2+t) .
$$

If in addition to (29) also,

$$
u_{0, x}-\left(\ln v_{0}\right)_{x x} \geq 0, \quad\left(\equiv w_{0, x} \geq 0\right),
$$

then (30) hold for any $\gamma \geq 3 / 2$. 
Proof. The Lemma is proved by imploying estimates of $[2,3]$. Let $\Phi=\Phi\left(v(t, x), V^{+}\right)$. One can easily compute using (7) and (3):

$$
\begin{aligned}
& \Phi_{t}=-\left(p(v)-p\left(V^{+}\right)\right) v_{t}=-\left(p(v)-p\left(V^{+}\right)\right) u_{x} \\
&=-\left(p(v)-p\left(V^{+}\right)\right)\left(u-u_{0}\right)_{x}-\left(p(v)-p\left(V^{+}\right)\right) u_{0, x} \\
&=-\left[\left(p(v)-p\left(V^{+}\right)\right)\left(u-u_{0}\right)\right]_{x}+\left(p(v)-p\left(V^{+}\right)\right)_{x}\left(u-u_{0}\right) \\
&-\left(p(v)-p\left(V^{+}\right)\right) u_{0, x} .
\end{aligned}
$$

Multiplying the second equation in (3) by $u-u_{0}$ we also get

$$
\begin{aligned}
\partial_{t} \frac{\left|u-u_{0}\right|^{2}}{2}-\left(\frac{1}{v} u_{x}\left(u-u_{0}\right)_{x}\right)_{x}+\frac{1}{v} u_{x}\left(u-u_{0}\right)_{x} & \\
& =-\left(p(v)-p\left(V^{+}\right)\right)_{x}\left(u-u_{0}\right) .
\end{aligned}
$$

On the other hand, from the first equation of (3) it follows that

$$
(\ln v)_{t}=\frac{1}{v} u_{x}
$$

Differentiating in $x$ and using the second equation of (3) we get

$$
\partial_{t} \frac{v_{x}}{v}=\partial_{x}\left(\frac{1}{v} u_{x}\right)=u_{t}+p(v)_{x}
$$

Multiplying this by $v_{x} / v$ we obtain

$$
\partial_{t}\left(\frac{1}{2}\left(\frac{v_{x}}{v}\right)^{2}\right)=\frac{p^{\prime}(v)}{v}\left|v_{x}\right|^{2}+\partial_{t}\left(\left(u-u_{0}\right) \frac{v_{x}}{v}\right)-\left(u-u_{0}\right) \partial_{t}\left(\frac{v_{x}}{v}\right) .
$$

Finally, using the previous equation in the last one we get

$$
\partial_{t}\left(\frac{1}{2}\left(\frac{v_{x}}{v}\right)^{2}+\frac{\left|u-u_{0}\right|^{2}}{2}-\left(u-u_{o}\right) \frac{v_{x}}{v}\right)-\frac{p^{\prime}(v)}{v}\left|v_{x}\right|^{2}=-\left(p(v)-p\left(V^{+}\right)\right)\left(u-u_{0}\right) .
$$

We add $2 \times(32)$, (33) and (34) and integrate the result in space over $\mathbb{R}$ and in time over $(0, t)$. Noticing that

$$
\begin{aligned}
& 2 \Phi+\left(\frac{v_{x}}{v}\right)^{2}+\frac{\left|u-u_{0}\right|^{2}}{2}-\left(u-u_{o}\right) \frac{v_{x}}{v} \leq 2\left(\Phi+\left(\frac{v_{x}}{v}\right)^{2}+\frac{\left|u-u_{0}\right|^{2}}{2}\right), \\
& 2 \Phi+\left(\frac{v_{x}}{v}\right)^{2}+\frac{\left|u-u_{0}\right|^{2}}{2}-\left(u-u_{o}\right) \frac{v_{x}}{v} \geq \frac{1}{4}\left(\Phi+\left(\frac{v_{x}}{v}\right)^{2}+\frac{\left|u-u_{0}\right|^{2}}{2}\right)
\end{aligned}
$$

and setting

$$
E_{1}(t)=\int \Phi\left(v(t, \cdot), V^{+}\right)+\left|\frac{v_{x}(t, \cdot)}{v(t, \cdot)}\right|^{2}+\frac{\left|u(t, \cdot)-u_{0}(t, \cdot)\right|^{2}}{2}
$$


we obtain:

$$
E_{1}(t)+\int\left(\frac{1}{2 v}\left|u_{x}\right|^{2}-\frac{p^{\prime}(v)}{v}\left|v_{x}\right|^{2}\right) \leq 2 \int \frac{\left|u_{0, x}\right|^{2}}{v}+\int\left(p\left(V^{+}\right)-p(v)\right) u_{0, x} .
$$

We consider first the case when $u_{0, x} \geq 0$ and $\gamma>2$.

In this equation, since $u_{0, x} \geq 0$ we get

$$
\int\left(p\left(V^{+}\right)-p(v)\right) u_{0, x} \leq 2 p\left(V^{+}\right) U^{+},
$$

since $u(t, \pm \infty)= \pm U^{+}$. Also,

$$
v^{-1} \leq c\left(\gamma, V^{+}\right) \Phi\left(v, V^{+}\right)^{\frac{1}{\gamma-1}}, \quad v<V^{+} / 2,
$$

and for $\gamma>2$, by Hölder inequality,

$$
\begin{aligned}
\int \frac{\left|u_{0, x}\right|^{2}}{v} \leq \frac{2}{V^{+}} \int_{v>V^{+} / 2}\left|u_{0, x}\right|^{2} & +\int \Phi^{\frac{1}{\gamma-1}}\left|u_{0, x}\right|^{2} \\
& \leq c\left(\gamma, V^{+},\left|u_{0, x}\right|_{\left.L^{2},\left|u_{0, x}\right|_{L^{\frac{2(\gamma-1)}{\gamma-2}}}\right)}\left(1+\left(\int \Phi\right)^{\frac{1}{\gamma-1}}\right) .\right.
\end{aligned}
$$

Thus,

$$
E_{1}(t) \leq E_{1}(0)+c t+\int_{0}^{t}\left(\int \Phi\right)^{\frac{1}{\gamma-1}}
$$

In particular,

$$
\int \Phi\left(v(t, \cdot), V^{+}\right) \leq E_{1}(0)+c t+\int_{0}^{t}\left(\int \Phi\right)^{\frac{1}{\gamma-1}} .
$$

The Gronwall type argument applies to the last inequality and we conclude that

$$
\int_{0}^{t}\left(\int \Phi\left(v(t, \cdot), V^{+}\right)\right)^{\frac{1}{\gamma-1}} \leq \leq c\left(1+E_{1}(0)+t^{\frac{\gamma-1}{\gamma-2}}\right)
$$

and consequently,

$$
E_{1}(t) \leq c\left(1+E_{1}(0)+t^{\frac{\gamma-1}{\gamma-2}}\right) .
$$

Following [3] we introduce a function

$$
\Psi(v)=\int_{V^{+}}^{v} \frac{\Phi\left(s, V^{+}\right)^{\frac{1}{2}}}{s} d s .
$$

It follows from the definition of $\Phi$ that

$$
v^{-\frac{\gamma-1}{2}} \leq c\left(\gamma, V^{+}\right)|\Psi(v)|, \quad 0<v<V^{+} / 2
$$

and

$$
v^{\frac{1}{2}} \leq c\left(\gamma, V^{+}\right)|\Psi(v)|, \quad v>2 V^{+}
$$


Also,

$$
\begin{aligned}
& |\Psi(v(t, x))| \leq \int\left|\Psi(v(t, x))_{x}\right| d x=\int\left|\frac{v_{x}}{v} \Phi\left(v, V^{+}\right)^{1 / 2}\right| d x \\
& \leq \int\left|\frac{v_{x}}{v}\right|^{2}+\int \Phi\left(v, V^{+}\right) \leq c\left(1+t^{\frac{\gamma-1}{\gamma-2}}\right),
\end{aligned}
$$

where in the last inequality we used (37). Using this result and (38) and (39) we obtain that

$$
\sup _{\mathbb{R}}|\ln v(t, \cdot)| \leq c \ln (2+t),
$$

for some $c$ independent of time. The first statement of the Lemma is proved.

Now, assume in addition that $w_{0, x} \geq 0$. Notice, that this condition follows from $u_{0, x} \geq 0$ if $v(0, x) \equiv$ const. We estimate term in the right hand side of (35) in the following way. As before, since $u_{0, x} \geq 0$ we get

$$
\int\left(p\left(V^{+}\right)-p(v)\right) u_{0, x} \leq 2 p\left(V^{+}\right) U^{+}
$$

Then,

$$
\int \frac{\left|u_{0, x}\right|^{2}}{v} \leq \frac{2}{V^{+}}\left|u_{0, x}\right|_{L^{2}}^{2}+\sup _{x: v(t, x)<V^{+} / 2} v(t, x)^{-1}\left|u_{0, x}\right|_{L^{2}}^{2} .
$$

Let

$$
\Psi_{1}(v)=\int_{V^{+}}^{v} \frac{\Phi\left(s, V^{+}\right)^{1 / 2}}{s^{(\gamma+2) / 2}} d s .
$$

It follows that there is a number $c\left(\gamma, V^{+}\right)>0$ such that

$$
v^{-\frac{2 \gamma-1}{2}} \leq c \Psi_{1}(v), \quad v<V^{+} / 2 .
$$

Then, since $-p^{\prime}(v) / v=c(\gamma) v^{-(\gamma+2)}$,

$$
\begin{aligned}
& \sup _{x: v(t, x)<V^{+} / 2} v(t, x)^{-1} \leq c \sup _{\mathbb{R}}\left|\Psi_{1}(v(t, \cdot))\right|^{\frac{2}{2 \gamma-1}} \leq\left|\int \frac{v_{x}}{v^{(\gamma+2) / 2}} \Phi\left(v, V^{+}\right)^{1 / 2}\right|^{\frac{2}{2 \gamma-1}} \\
& \leq c\left[-\epsilon \int \frac{p^{\prime}(v)}{v}\left|v_{x}\right|^{2}+c_{\epsilon} \int \Phi\left(v, V^{+}\right)\right]^{\frac{2}{2 \gamma-1}} \\
& \leq-c \epsilon \int \frac{p^{\prime}(v)}{v}\left|v_{x}\right|^{2}+c_{\epsilon}\left(1+\left(\int \Phi\left(v, V^{+}\right)\right)^{\frac{2}{2 \gamma-1}},\right.
\end{aligned}
$$

where the last inequality is possible for $2 /(2 \gamma-1) \leq 1$, i.e. $\gamma \geq 3 / 2$. Using the last result in (42) we obtain that

$$
\int \frac{\left|u_{0, x}\right|^{2}}{v} \leq-c \epsilon \int \frac{p^{\prime}(v)}{v}\left|v_{x}\right|^{2}+c_{\epsilon}\left(1+\left(\int \Phi\left(v, V^{+}\right)\right)^{\frac{2}{2 \gamma-1}}\right.
$$


with $c^{\prime} s$ independent of time. Using the last inequality and (41) in (35) and choosing $\epsilon$ suitably small we obtain that

$$
E_{1}(t) \leq E_{1}(0)+\int_{0}^{t} c\left(1+\left(\int \Phi\left(v(\tau, \cdot), V^{+}\right)\right)^{\frac{2}{2 \gamma-1}} d \tau\right.
$$

Now, we obtain an estimate on $\int \Phi\left(v, V^{+}\right)$. Arguing in the same way as in (32) we get

$$
\begin{array}{r}
\Phi\left(v, V^{+}\right)_{t}=-\left[\left(p(v)-p\left(V^{+}\right)\right)\left(w-w_{0}\right)\right]_{x}+\left(w-w_{0}\right)\left(p(v)-p\left(V^{+}\right)\right)_{x}-\left(p(v)-p\left(V^{+}\right)\right) w_{0, x} \\
-\left[\frac{v_{x}}{v}\left(p(v)-p\left(V^{+}\right)\right)\right]_{x}+\frac{p^{\prime}(v)}{v}\left|v_{x}\right|^{2} .
\end{array}
$$

From the second equation in (5) we get

$$
\partial_{t} \frac{\left|w-w_{0}\right|^{2}}{2}=-\left(w-w_{0}\right)\left(p(v)-p\left(V^{+}\right)\right)_{x}
$$

Adding the last two equations and integrating in $x$ over $\mathbb{R}$ we get

$$
\partial_{t} \int \Phi\left(v(t, \cdot), V^{+}\right)+\frac{\left|w(t, \cdot)-w_{0}(\cdot)\right|^{2}}{2}-\int \frac{p^{\prime}(v)}{v}\left|v_{x}\right|^{2}=\int\left(p\left(V^{+}\right)-p(v)\right) w_{0, x} .
$$

Now, since $w_{0, x} \geq 0$ we have $\int\left(p\left(V^{+}\right)-p(v)\right) w_{0, x} \leq 2 p\left(V^{+}\right) U^{+}$, since $w(t, \pm \infty)= \pm U^{+}$. Integrating the above inequality in time we obtain

$$
\int \Phi\left(v(t, \cdot), V^{+}\right) \leq c(1+t) .
$$

Note, that this is the best possible estimate for the left hand side with respect to the growth rate in time, since we expect that $v$ converges to $V^{e}$. In any case, using this estimate in (44) we obtain that

$$
E_{1}(t) \leq E_{1}(0)+c\left(1+t^{2}\right)
$$

The second statement of the Lemma follows by the same argument we used above, see (37) and below.

Combining Lemma 24 and Lemma 25 we get

Corollary 1. For any $t>t_{0}>0$ there is $c$ independent of $t$ such that

$$
\begin{gathered}
\int\left(\Phi\left(v(t, \cdot), V^{e}(t, \cdot)\right)+\frac{\left|w(t, \cdot)-U^{e}(t, \cdot)\right|^{2}}{2}\right) \\
-\int_{t_{0}}^{t} \int \frac{p^{\prime}(v)}{v}\left|v_{x}\right|^{2} \leq \int\left(\Phi\left(v\left(t_{0}, \cdot\right), V^{e}\left(t_{0}, \cdot\right)\right)+\frac{\left|w\left(t_{0}, \cdot\right)-U^{e}\left(t_{0}, \cdot\right)\right|^{2}}{2}\right) \\
+c(\ln (2+t))^{2} .
\end{gathered}
$$


Proof. We estimate the last term appearing in the inequality of Lemma 1. Note, that

$$
\begin{gathered}
p\left(V^{e}\right)_{x}=c \frac{(x / t)^{\frac{\gamma-1}{\gamma+1}}}{t} \rightarrow 0, \quad x \rightarrow 0 \\
p\left(V^{e}\right)_{x}=0, \quad|x|>\xi^{+} t
\end{gathered}
$$

and

$$
p\left(V^{e}\right)_{x x}=c \frac{(x / t)^{-\frac{2}{\gamma+1}}}{t^{2}}, \quad|x|<\xi^{+} t .
$$

We can write by integrating by parts:

$$
\begin{gathered}
\left|\int_{t_{0}}^{t} \int \frac{v_{x}}{v} p\left(V^{e}\right)_{x}\right|=\left|\int_{t_{0}}^{t} \int_{|x|<\xi^{+} t}(\ln v)_{x} p\left(V^{e}\right)_{x}\right| \\
=\left|\int_{t_{0}}^{t}(\ln v) p\left(V^{e}\right)_{x}\right|_{x=0}^{x=\xi t}+\left.(\ln v) p\left(V^{e}\right)_{x}\right|_{x=-\xi t} ^{x=0}+\int(\ln v) p\left(V^{e}\right)_{x x} \mid \\
\leq c \int_{t_{0}}^{t}\left(\sup _{\mathbb{R}}|\ln v(t, \cdot)|\left(1 / t+c \int_{0}^{|x|<\xi^{+} t}(x / t)^{-\frac{2}{\gamma+1}} \frac{1}{t^{2}} d x\right)\right. \\
\leq c \int_{t_{0}}^{t} \frac{\sup _{\mathbb{R}}|\ln v(t, \cdot)|}{t} \leq c(\ln (2+t))^{2},
\end{gathered}
$$

where in the last inequality we used Lemma 2.

Corollary 2. Let $v^{t}(y)=v(t, t y), w^{t}(y)=w(t, t y)$ and $u^{t}(y)=u(t, t y)$. Then,

$$
\begin{array}{r}
\int \Phi\left(v^{t}(y), V^{e}(y)\right)+\left|w^{t}(y)-U^{e}(y)\right|^{2} d y \rightarrow 0, \quad t \rightarrow \infty, \\
u^{t}(\cdot) \rightarrow U^{e}(\cdot), \quad t \rightarrow \infty, \text { in } H^{-1}\left(B_{R}\right) \\
\forall \epsilon>0, \quad \text { meas }\left((t, \infty) \cap\left\{t: \int \frac{\left|u^{t}-w^{t}\right|^{2}}{\left(v^{t}\right)^{\gamma+1}} d y>\epsilon\right\}\right) \rightarrow 0, \quad t \rightarrow \infty,
\end{array}
$$

where $B_{R}$ is a ball of an arbitrary radius $R$.

Proof. The first statement follows immediately from the previous corollary by the change of variables $x \rightarrow t y$ in the first integral on the left hand side of the inequality. To prove the second, we notice that from (3) and (5) it follows that

$$
u(t, x)-w(t, x)=(\ln v(t, x))_{x} .
$$

Take a $\psi(y) \in C_{0}^{\infty}\left(B_{R}\right)$ and use the above equation to estimate:

$$
\begin{aligned}
\left|\int\left(u^{t}(y)-U^{e}(y)\right) \psi(y)\right|=\mid \int\left(w^{t}(y)\right. & \left.-U^{e}(y)\right) \psi(y)-\frac{1}{t} \int \ln v^{t}(y) \psi^{\prime}(y) \mid \\
& \leq c\left(\left|w^{t}-U^{e}(y)\right|_{L^{2}}+\frac{\ln (2+t)}{t}\right)|\psi|_{H^{1}\left(B_{R}\right)},
\end{aligned}
$$


where in the last inequality we used the estimate of Lemma 2. The second statement is proved as well.

From (51) we also deduce that

$$
\int \frac{|u(t, x)-w(t, x)|^{2}}{v(t, x)^{\gamma+1}}=\int \frac{\left|v_{x}\right|^{2}}{v^{\gamma+2}}=-c \int \frac{p^{\prime}(v)}{v}\left|v_{x}\right|^{2}, \quad c=c(\gamma)>0 .
$$

Integrating in time and using Corollary 1 we get

$$
\int_{t_{0}}^{t} \tau \int \frac{\left|u^{t}-w^{t}\right|^{2}}{\left(v^{\tau}\right)^{\gamma+1}} d \tau \leq c\left(t_{0}\right)+c(\ln (2+t))^{2} .
$$

The third statement follows from here.

\subsection{Appendix: Rarefaction waves for Euler equations}

Here we recall the construction of rarefaction waves for Euler equations. We are solving problem (1), (6). For simplicity we assume that the initial data and the solution have the following symmetry:

$$
v(t,-x)=v(t, x), \quad u(t,-x)=-u(t, x)
$$

Setting

$$
v(t, x)=V(x / t), \quad u(t, x)=U(x / t),
$$

we obtain from the Euler equations

$$
\left[\begin{array}{cc}
-\xi & -1 \\
-\gamma V^{-\gamma-1} & -\xi
\end{array}\right] \cdot\left[\begin{array}{c}
V^{\prime} \\
U^{\prime}
\end{array}\right]=0
$$

From which we obtain:

$$
\xi= \pm\left(\gamma V^{-\gamma-1}\right)^{1 / 2}
$$

and an ODE:

$$
\xi V^{\prime}+U^{\prime}=0
$$

which we can solve using (53). For that, let us fix a right state $\left(V^{+}, U^{+}\right)$, set $\xi^{+}=$ $\left(\gamma\left(V^{+}\right)^{-\gamma-1}\right)^{1 / 2}$ and integrate the ODE.

$$
U=U^{+}+\frac{2 \gamma^{1 / 2}}{\gamma-1}\left[V^{\frac{1-\gamma}{2}}-\left(V^{+}\right)^{\frac{1-\gamma}{2}}\right]
$$

Since $\xi<\xi^{+}$we have $V>V^{+}$and $U<U^{+}$. Moreover, if

$$
U_{0}:=U^{+}-\frac{2 \gamma^{1 / 2}}{\gamma-1}\left(V^{+}\right)^{\frac{1-\gamma}{2}}>0
$$

then, as $\xi \rightarrow 0,(V(\xi))^{-1}$ rarefies to 0 and $U(\xi)$ decreases to $U_{0}$ and vacuum region appears. 
Summarizing, for $\xi=x / t \neq 0$ the solution $\left(V^{e}, U^{e}\right)$ is defined as:

$$
\left\{\begin{array}{cc}
V^{e}(\xi)=V^{+}, U^{e}(\xi)=U^{+} & |\xi|>\xi^{+}, \\
V^{e}(\xi)=\left(\frac{\gamma}{\xi^{2}}\right)^{\frac{1}{\gamma+1}} & 0<|\xi|<\xi^{+}, \\
U^{e}(\xi)=U^{+}+\frac{2 \gamma^{1 / 2}}{\gamma-1}\left[V^{e \frac{1-\gamma}{2}}-\left(V^{+}\right)^{\frac{1-\gamma}{2}}\right] & 0<|\xi|<\xi^{+}, \\
\xi=\left(\gamma\left(V^{+}\right)^{-\gamma-1}\right)^{1 / 2} &
\end{array}\right.
$$

Moreover, since $u(t, x) \rightarrow \pm U_{0}$ as $x \rightarrow 0 \pm$, we conclude that $v(t, 0)=2 U_{0} t \delta_{0}(x)$.

\section{References}

[1] D. Hoff, Global solutions of the equations of one-dimensional, compressible flow with large data and forces, and with differing end states, Z. Angew. Math. Phys. 49(1998), no. 5, p.774-785.

[2] A. Matsumura and K. Nishihara, Asymptotics toward the Rarefaction Waves of the Solutions of a One-Dimensional Model System for Compressible Viscous Gas, Japan J. Appl. Math. , 3(1986),p.1-13.

[3] H. Y. Wang, Zero Dissipation Limit to Rarefaction Waves for the $p$-System, Acta Math. Sinica, 21 Vol. 21(2005), p.1229-1240. 